\title{
Cagliostro in Straßburg nach der Schilderung eines Augenzeugen.
}

Von HEINRICH FUNCK.

Zu den merkwürdigsten Erscheinungen des 18. Jahrhunderts, des Zeitalters der Aufklärung, gehört das Auftreten des Wundergrafen Cagliostro. An keinem Ort, nicht in Petersburg, nicht in Berlin, auch nicht in Paris hat dieser blendende Abenteurer großen Stils, der noch heute an Originalität, List und Unverschämtheit alle seine zahlreichen Nacheiferer in Schatten stellt, seine Rolle so glänzend und so ungestört spielen können, wie in Straßburg, wo er am 27. September 1780 seinen Einzug hielt. Franzosen und Deutsche wetteiferten in der elsässischen Metropole in Bewunderung und Vergötterung des geheimnisvollen Fremdlings, der sich Graf Alessandro di Cagliostro, Schüler des weisen Althotas, Pflegesohn des Scheriffs von Mekka und dergleichen mehr nannte, von Profession Heiler von Krankheiten, Entferner von Runzeln, Helfer der Armen und Impotenten, Großmeister der ägyptischen Loge zur hohen Wissenschaft, Geisterbeschwörer, Goldmacher, Großkophta, Prophet, Taschenspieler, kurz ein König der Schwindler und Lügner war, und verbreiteten den Ruhm seiner sibyllinischen Weisheit in alle Welt. Im Verhältnis zu der Berühmtheit aber, welche der Charlatan der Charlatane in der alten Reichsstadt erlangte, sind nur sehr wenige authentische Nachrichten von seiten derer, die hier mit ihm verkehrt haben, auf uns gekommen. Eine Hauptquelle bieten die 1853 in Paris erschienenen Denkwürdigkeiten der Baronin Henriette Luise von Oberkirch, geborene von Waldner, die mit ihrem Gatten Ende Oktober 1780 nach StraBburg kam und dort bei dem Fürstbischof und Kardinal Louis von Rohan, dem nachmaligen Helden der Halsbandgeschichte, den angeblichen Grafen kennen lernte. 
Allein man weiß nicht, inwieweit der geistreichen Dame der Drang, die Wahrheit zu enthüllen, oder die Lust, ihre Leser angenehm zu unterhalten, die Feder führte.

Nur wenig ungedrucktes Quellenmaterial stand Louis Spach (Oeuvres choisies V. 1871) bei seiner Darstellung des Lebens und Treibens der Straßburger Gesellschaft während der Anwesenheit des Wundermannes zu Gebote. Vier neue Briefe über Cagliostro in Straßburg wurden von Hans von ZwiedineckSüdenhorst aus einem alt-gräflichen Familienarchiv ans Licht gezogen. (Siehe Beilage zur Allgemeinen Zeitung 1894, Nr. 64, 65,67 .) Eine weitere briefliche Äußerung eines Augen- und Ohrenzeugen, die sich in Bodmers handschriftlichem Nachlaß in Zürich erhalten hat, soll auf den folgenden Blättern zum Abdruck gelangen. Unser Gewährsmann ist der Zunftmeister und Dichter Johannes Bürkli aus Zürich, der im Herbste 1781 mit seiner Frau nach Straßburg reiste, um Cagliostro daselbst zu konsultieren. Unterwegs traf er in Brugg mit dem Züricher "Propheten" Lavater zusammen, der eben bei seinem Freund Jacob Sarasin in Basel den weltberühmten Heilkünstler gesehen und gesprochen hatte. Wir lesen in Lavaters Tagebuch 1781: „22. Oktober. Auf Basel, Sarasin und Cagliostro da. Der Frau Sarasin wurde durch Cagliostro geholfen 1) von Gichtern, 2) Schlaflosigkeit, 3) Frieren, 4) Mangel an Appetit. Cagliostro gewiß ein außerordentlicher Mensch, aber erstaunlich stolz, schnell aufgebracht, viel Prätension auf magische Einflüsse und Erfahrungen. 24. Oktober. Wieder zurück bis Brugg zum Übernachten, Bürkli und Frau angetroffen."

In Straßburg schlossen sich Bürklis an das ehrenfeste Sarasinsche Ehepaar an, das mit dem großen Schwindler schon geraume Zeit eng liiert war. Gertrud Sarasin, geborene Battier, war seit April 1781 in Cagliostros Behandlung. Die effolgreiche Wunderkur, die dieser neue Gott der Arzneikunde mit ihr vornahm, hat der überglückliche dankbare Gatte in einem Schreiben vom 10. November 1781, das Kardinal von Rohan als Reklame für seinen Schützling im Journal de Paris veröffentlichen ließ, ausführlich beschrieben. Vom 28. Oktober 1781 bis 18. September 1782 wohnte Jakob Sarasin aus Basel mit seiner ganzen 
Familie dem Retter seiner Frau zuliebe in Straßburg. Er notiert in seinem Tagebuch 1781: „13. November. Nachmittags Visite vom Grafen und Bürkli. 17. November. Morgens beim Grafen, nachmittags bei Bürkli. 27. November. Nachmittags bei Bürkli.“

Nachdem Johannes Bürkli bald ein Vierteljahr Gelegenheit gehabt hatte, Cagliostros Wirksamkeit aus nächster Nähe zu beobachten, unternahm er es, ein naturgetreues Bild davon seinem alten Lehrer Bodmer $z \mathfrak{u}$ entwerfen. Die "dicke Epistel * ist "Straßburg vom 14 ten bis 17 ten Jenner 1782" datiert und lautet, soweit sie von Cagliostro handelt, folgendermaßen:

$\mathrm{Da}$ ich Ihnen von Lavatern rede, verehrungswürdiger Greis, so führt mich meine natürliche Ideenverbindung auf unsern medicinischchymischen Lavater in Straßburg, den berühmten Grafen von Calliostro, der den Stein der Weisen ebenso zuverlässig gefunden, als Lavater unsere Beschäftigungen und Vergnügungen in der Ewrigkeit mit seinem Seherauge entdeckt haben soll.1) $\mathrm{Da}$ ich Ihnen von keinem wunderseltsammern Phenomen aus Straßburg etwas melden kann, so erlauben Sie mir, verehrungswürdiger Greis, daB ich Sie gleich auf einige Augenblike von diesem unterhalte, um so vielmehr, da ich mir seit meinem hiesigen Aufenthalt Mühe gegeben, dieses Wunderding in der Nähe zu beobachten. Dieser Mann, der heüt zu Tage in unserm winzigen Weltwinkel mehr Lerm als kein Trompeter und Paukenschläger macht, langte im Christmonat $\left.1779,{ }^{2}\right)$ ohne einiges Gefolge, ohne einige Empfehlung mit seiner Frau (oder Maitresse, der Himmel mag wissen, welche Stelle sie bekleidet) aus RuBland und Pohlen hier in StraBburg an, hielt sich acht bis zehen Tage ganz in seinem Zimmer verschlossen und einsam, wandte sich zuerst an die hiesigen Freymäürer, von denen auch er Ordensbruder ist, prangte mit berühmten Namen großer Pohlnischer und Russischer Herren, die er seine innigsten Freünde und Vertraute nennte. Bey genauerer Untersuchung fand es sich, daß beynah alle diese würklich große Herren, was der Franzose im moralischen Sinn des Roués nennt, waren. Hierauf äüBerte er gegen einige hiesige Bürger, die ihn zum erstenmale besuchten, das Verlangen, daß seine Frau in den hiesigen Gesellschaften möchte eingeführt werden, welches einige der gescheidesten von ihm sogleich entfernte. Sogleich änderte er die Larve, schaffte sich eine prächtige Equipage an, ließ Demanten und Solitaires an seinen Fingern glänzen, dingte drey hübsche wohlgewachsene Bediente, die er in grüne Livree reich mit Silber verbrämt steken ließ, miethete sich ein geräümiges Zimmer mit damastenen Vorhängen, reich vergüldeten Listren, Crystallleüchtern und

1) Anspielung auf Lavaters vielgelesenes Buch "Aussichten in die Ewigkeit". nach StraBburg.

2) Einer Notiz in Sarasins Tagebuch zufolge kam Cagliostro am 27. September 1780 
Geräthe vom neüsten Geschmak und größten Wehrte, ließ sich bey Herrn Marquis de la Salle, Commendanten der Provinz, Mr. de la Galaisière, Intendanten, Mr. de l'Or, Lieutenant du Roi, und beym Cardinal von Rohan, hiesigen Bischoff, als Graf von Calliostro melden, gab Ihnen geschikt und arglistig zu verstehen, daß unter dem bescheidenen Titul eines Grafen wol etwas höheres, das aber die wichtigsten politischen Beweggründe ihn zu entdeken hinderten, verborgen wäre, sprach viel von den Sitten aller vier Welttheile, die er durchwandert hätte, von seiner Vertraulichkeit mit der Russischen Kayserinn, dem König von Pohlen, dem Groß Sultan und vorzüglich mit seinen Sultaninnen, in deren Serail er wie bey Hause wäre und sie sans façon, wie unser einer seine nächsten Verwandten, Morgens am Puztisch, Nachmittags, Abends auch wol gar bisweilen im Bade, im Nachtgewande und im vertraulichsten Tête à Tête besuchte. Maul und Augen wurden da, wie billig, aufgesperrt, die Hände übern Kopf zusammengeschlagen, und die Ah, Ah!!! mit nachlaufenden Interjektionspunkten wälzten sich einmal über das andere über die Lippen, während dem einige gescheidte Männer, die ich kenne, und die zwar nicht Ärzte, aber auch nicht angebrannte Köpfe sind, lächelten und sich leise wegschlichen. Was die Bewunderung und Achtung für den Mann noch mehr vergrößerte, war, daß er sich als einen der größten Chymisten unsres Zeitalters ausgab und die einen versicherte, den Stein der Weisen gefunden zu haben. Bey andern rühmte er sich, das beneidenswehrte Arcanum zu besitzen, alte entnervte Titons ${ }^{1)} \mathrm{zu}$ verjüngern und ihnen $z \mathfrak{u}$ Cytherens Dienst neüe Kräfte zu schenken, die ihren lodernden Begierden angemessen wären. Auch soll er würklich in diesem Fach einige nicht unglükliche Versuche gewagt haben, und in seiner Geschiklichkeit in ersterer Wissenschaft scheint er selber der größte Beweis, da er seit mehr als zwey Jahren, daß er hier wohnt und Equipage und drey Bediente hält, Bälle, diners und soupers seinen Patienten giebt, von keinem Banquier in Europa, so viel man zuverlässig weiB, weder Geld noch Wechsel empfangen, nichts von seinem Geräthe verkauft und auch keinen Heller Schulden gemacht hat. Ist sichs nun zu verwundern, daß ein Mann, der die Kunst Gold zu machen und die erstorbne Natur zu beleben - d. i. Zwek und Mittel die Freüden des Lebens zu genießen, zu verschaffen verspricht, bey den Großen der Erde, die nach beydem so heißhungrig jagen, so leichten und freyen Zutritt findet, sollt' er auch von Galgen und Rade heruntergefallen seyn, und warum sollte man auch seinen Worten nicht glauben, kann doch niemand besser wissen, welche Zauberkünste er besizet, als er selber!

DiB waren seine Neze, den vornehmen Pöbel in Straßburg zu verstriken. Ganz andere warf er für den gemeinen Pöbel aus. Rings um

1) Tithonos, der Gemahl der Eos, dem ewiges Leben, aber nicht ewige Jugend von den Gōttern geschenkt wurde, so daB seine Glieder austrockneten. 
sich her versammelte er Krüppel, Tropfschlägige, Taube, Gichtbrüchige u. s. w. heilte die einen, sandte die andern mit guten Empfehlungsschreiben in Plutons Schattenreich, den Vornehmen gab er seine Consulten und Besuche umsonst, den Armen auch so gar seine Arzneyen nicht selten gab er ihnen noch reiche Allmosen dazu, sich desto besser verpflegen und gütlich thun zu können. Von früh 6 Uhr des Morgens im Winter, 5 Uhr im Sommer, bis spät Abends um 9 oder $10 \mathrm{Uhr}$, rasselte er in seinem Wagen durch Stadt und Vorstädte, und öfters noch sah man ihn vor der hölzernen, Einsturz drohenden Hütte des Schusters und Bürstenbinders als vor dem pralenden Hotel des Reichen stille halten - daher auch immer sein Wagen von einem zahlreichen Trupp Gesindel, Männern, Weibern, Kindern, (wie unser theologische Calliostro Lavater in Augspurg) $)^{\text {) }}$ verfolget war. Einige warfen sich auf die Knie, andere beteten für ihn Rosenkränze, andere machten Kreüze, andere besprengten den Wagen mit Weyhwasser, und rechts und links flogen aus dem Wagen kleine Liebessteüern. Wenig hätte gefehlt, so wäre die Zahl der Heiligen mit einem Sankt Calliostro vermehrt worden.

Meinem Helden giengs auch in Straßburg, wie Boileau sagt, daß es ihm in Paris gegangen. Seine Feinde und Neider nüzten ihm mehr als seine enthusiastischen Verehrer und Freünde. Die Rotte der hiesigen Ärzte durch ihre Ränke, Verleümdungen, übeln Nachreden, schmükten sein Haupt mit neüen Loorbeeren und fesselten sich selber ohne ihr Wissen und wider ihren Willen an seinen Triumphwagen. Just auf der Seite griffen sie ihn an, wo seine große Stärke lag. Sie suchten seine Uneigennüzigkeit verdächtig zu machen, und die ganze Stadt stand wider sie auf zu Zeügen. Sie behaupteten mit eherner Stirne, daß Calliostro ein ganz gewöhnlicher Marktschreyer, ohne einen Schatten von Theorie seiner Kunst, ohne einige Kenntniß der Anatomie und Botanik wäre, und alle Tage strafte sie eine neüe Wunderkur, wie die von Herrn La Salle Sekretair Le Monnier, von Herrn Langlois, L'Officier von Colmar, von der Mad. Sarasin von Basel u. s. w. Lügen. Denn tiefe Kenntniß wird kein vernünftiger und unpartheyischer Mann, der sich drey Monate lang in Straßburg aufgehalten hat, Calliostro absprechen, so ungern ich ihm neben seiner Kunst vorzügliche Verdienste zugestehen möchte. Diese medecinische Fehde trug den Namen Calliostro weit über Straßburgs Mauern hinaus und lokte einen Schwarm von würklichen und eingebildeten Kranken, von Dames à Vapeurs, von schwachnervigten süßen Herrchens u. s. w. aus der Hauptstadt, von Marseille, Lyon u. s. w. Gerade diß wars, was Calliostro gesucht hatte, und diesen Dienst, den ihm seine glühenden Freünde mit allem Trompeten- und Paukenschalle in Journalen, Zeitungsblättern u. s. w. in ihren von Weyhrauch triefenden Versen und Madrigaux

1) Am 21./22. Juni 1778 auf der Rückreise von Pondorf bei Straubing, wo er den Teufelsbanner Gaßner aufsuchte. 
nicht hätten bewürken können, leisteten ihm seine Feinde. Nun hatte Mad. Calliostro auf ihrem Zimmer eine Hofhaltung so gut als Mad. la Marquise de la Salle und Mad. de la Galaisière - nur mit dem Unterschied: diese leztern hielten wöchentlich nur zweymal Cour, Mad. Calljostro alle Abende - bey jenen währten die Besuche höchstens eine halbe Stunde, bey dieser von 5 bis 8 Uhr. In einem kleinen Zimmer machten die Damen einen Creis um Mad. Calliostro, die ihre Ecke beym Caminfeüer (La Place d'Honneur) gegen jedermann treülich behauptete. Im Vorzimmer (einem großen geräümigen Saal!) empfing der Graf auch am Camin die fremden Gäste. Die Unterredung in beyden Zimmern war gewöhnlich von den Krankheiten, die jeder Zeitlebens gehabt, noch habe, oder in Zukunft haben möchte und würde - von den Wundercuren des Grafen. Mad. Sarasin von Basel, als Dechantinn, spielte bey dieser Farce die Hauptrolle, widerholte jedem neüen Ankömmling die abentheüerliche Geschichte ihrer Krankheit und Genesung, wie sie Calliostro mit seinem Zauberstabe dem Tod unter den Zähnen aus offenem Schlunde weggerissen hätte, wie ihre, dem Himmel sei Dank, gesegneten Leibesumstände, das Weiches zu erweisen war, von Calliostros Heilkunst wären u. s. w. ${ }^{1}$ ) Ein zweytes Wunderwerk ward an einem verjährten, seit dem 7 ten Jahre tauben gnädigen Fräülein aufgestellt, der Calliostro das Gehör widergeschenkt haben sollte, obgleich auf jede Frage, die man an sie that, noch 10 Plaît-il? Comment Monsieur? Je ne Vous entends pas u. s. w. folgten. Dieses soll, wie man sagt, izt nur noch zurükgebliebne schlimme Gewohnheit seyn. Eine Parisische Spielerinn, deren Wannst einen völligen halben Mond ausmachte, welches sie gewaltig hinderte, die Karten zum Gesichte zu bringen und mit Grâce und einer gewissen Noblesse und Décence zu spielen, sollte Calliostro ihrer überflüssigen Fettigkeit, mit der man manches Brachfeld in unserm lieben Vaterland hätte düngen können, entladen. Weil sie sich aber seiner vorgeschriebenen Diät nicht unterwerfen und weniger als täglich acht Pfund Fleisch, ohne Entremets und Légumes zu rechnen, zu essen verstehen wollte, so reiste sie wieder unverrichteter Dinge nach Paris zurük. Nicht selten traf man ehmals in diesen Cercles Herrn de la Salle, de la Galaisière, de l'Or, auch wol gar den Cardinal de Rohan, Herrn von Flachsland (dritten Commendanten) an, die ihres Ranges nicht für unwürdig hielten, die Rolle von Calliostros Apothekerjungens zu spielen. Denn da Calliostro kein einiges Recept eigenhändig verschreibt, so leisteten ihm diese Männer treüe Dienste, und wenn irgend ein Patient ihn raths fragte, klopfte Calliostro dem ersten besten dieser Herren, der neben ihm stand, vertraulich auf die Schulter mit dem Complimente Mon Ami de la Salle - mon Ami de Rohan - Mon Ami de la Galaisière 1. s. w. écrivés ce que je vais Vous

1) Den 4. April 1782 kam Frau Sarasin in Straßburg mit einem Knaben nieder. Sarasins Tagebuch 1782: "4. Apr. Um 5 Uhr 20 Minuten ein Sohn zur Welt. Cagliostro da. - 5. Apr. Nachmittags Taufe meines Sohnes Alexander (zu Ehren Cagliostros) Jakob. Graf und Gräfin gegenwärtig.“ 
dicter - und siehe sie schrieben, so gut sie konnten, und Calliostro übersah und verbesserte ihr Argument. Freylich haben sich izt seit ein paar Monaten die Zeiten und Sitten für Calliostro verschlimmert, weder de la Salle noch Galaisière erscheinen mehr bey ihm, sondern drey bloße Privatmänner Mr. de Barbier, Herr Straub ${ }^{1}$ ) und Herr Sarasin von Basel bekleiden gegenwärtig bey ihm Sekretairs und Apothekerjungens Stelle.

Man hat hier zu beobachten geglaubt, daß seit der Zeit da Calliostro mit dem Cardinal in so enge Vertraulichkeit getretten, daß er ihm beynahe unentbehrlich geworden ist und sich 14 Tage, 3 Wochen und noch länger in seinem Lustschloß zu Saverne aufhält, seit dem er sichs angewöhnt hat, bey Herrn de la Salle, de la Galaisière u. s. w. in freündschaftlichem Tête à Tête zu Mittag und zu Nacht zu speisen, seitdem Mad. Sarasin mit ihrer ganzen Familie sich hier häuslich niedergelassen, Calliostros Menschenliebe und GroBmuth gegen die Armen ein wenig in Schwindsucht gefallen, nicht daß er Ihnen nicht immer noch Rathschläge und Arzneyen unentgeldlich ertheile, allein statt in alle diese ekelhaften Hütten des Elends persönlich hinzufahren, hat er nun wöchentlich einmal einen allgemeinen Audienztag ausgesezt, Freytag Morgens von 11 Uhr bis halb ein Uhr. $\mathrm{Zu}$ denen, die Krankheit halber nicht selber $\mathrm{zu}$ ihm hingehen können, schikt er einen seiner dienstbaren Geister, Namens Jaquaut, der vorher Commis beym hiesigen Kaufhause war und von der Arzneykunst ungefehr so viel, als ich verstehen mag. Auf dessen Bericht kommt nun Krankheit oder Genesung, Leben oder Tod seiner Patienten an. Dieser theilt auf Calliostros Verordnung Arzneyen aus. Aus eigner Erfahrung glaub' ich kühn behaupten zu dürfen, daß izt er die Reichen den Wehrt seiner Arzneyen wenigstens gedoppelt bezahlen läßt, um sie den Armen desto leichter unentgeldlich geben zu können - Eine Bescheidenheit dieses großen Mannes, um den allzu blendenden Glanz seiner Großmuth für blöde Augen zu mildern! Zuverlässig glaub' ich aus vielen Thatsachen behaupten zu dürfen, daB bey ihm brennender Ehrgeiz die Stelle des Eigennuzes vertrittet, in dem er nur diejenigen (!) Patienten, die von allen übrigen Ärzten verlassen worden und mit verzweifelten unheilbaren Übeln behaftet zu seyn glauben, alle seine Muße, Kräfte und Sorge widmet, hingegen andere von gewöhnlichen Nervenkrankheiten, Magenbeschwerden, Fieber u. s. w. schändlich vernachlässigt. So wahr ist Helvetius Grundsaz Que l'Intérêt propre est le mobile de toutes nos Actions, und Rochefaucaults Maxime: Que nos Vertus les plus brillantes ne sont le plus souvent que des Vices déguisés. - Auch fährt er heut zu Tage ganz einsam und vom Pöbel ebenso unbegafft, als meine eigene Wenigkeit, durch die Straßen, und La Salle, Galaisière, de l'Or, Flachs-

1) An ihn ist die eingangs von mir erwähnte "Lettre de M. Sarasin, Négociant de Bâle, à M. Straub, Directeur de la Manufacture Royale d'Armes blanches en Alsace, Strasbourg le 10 Nov. 1781" gerichtet, die im Supplément au No. 365 du Joumal de Paris, Lundi 31. Décembre 1781 erschien. - Vgl. Sarasins Tagebuch 1781: „7. Nov. Brief an Straub angefertigt für den Cardinal." 
land werden nicht mehr in seinen Zimmern erblikt. Bald, bald wird (mit unserm FüeBli zu reden) auch dieser Dunst den Weg aller Dünste gehen - Le Masque tombe, l'Homme reste, et le Héros s'évanouit. Nun, verehrungswürdiger Greis, da ich Ihnen die Geschichte dieses wunderbaren Mannes, theils aus eigener Beobachtung, theils aus dem Munde von mehr als 20 Zeügen, worunter gewiß Männer von Gewichte sind, deren bloße Namen schon der Sache Nachdruk gäben, wenn nicht Citationen immer odiosa wären, erzählt habe, so erlauben Sie mir noch Ihnen meine eigene Herzensgesinnung über ihn und die Eindrüke, die er auf mich gemacht hat, in ihren Schoos auszuschütten. Auf meiner Reise von Zürich nach StraBburg spies ich durch Zufall in Bruk mit unserm Lavater und Schweizern, die um Calliostro zu sehen und zu sprechen expreß die Reise von Zürich nach Basel gemacht, wo er sich damals bey Herr Sarasin aufhielt, zu Nacht. Ein großer Theil der Unterrednung war von Calliostro. Aufmerksam hört' ich zu und machte mirs zur Pflicht, sobald ich in Straßburg anlangte, vor allem anderm aus dieses Phenomen zu beobachten. Auf der einen Seite verschanzt ich mich ebenso wol gegen den glühenden Enthusiasmus des Geistersehers als gegen die Härtigkeit und Zweifelsucht meines eignen Herzens - denn Sie wissen, ich bin in diesem Punkt ein wenig Britte, et je n'admire rien. Wenige Tage nach meiner Ankunft hier besucht ich ihn mit meiner Frau des Abends, unter der Egide seiner Favoritinn, der Mad. Sarasin. Sein erster Anblik stürzte mich ein wenig aus den Wolken, in die sich meine Einbildungskraft, durch Lavatern aufgedunsen, verstiegen hatte, herunter. Mein Gedächtni $\beta$ rief mir entfernte und dunkle Ähnlichkeiten zurük mit einem Camillo Mari (bey uns genannt der Türk), der, als ich in die lateinische Schule gieng, auf dem Weinplaz seine Bühne mit Hannswurst, Aff und Comp. aufgeschlagen hatte und da seinen Heil- und Wunderbalsam verkaufte und mit andern Herrn solches Gelichters. In seiner ganzen Physiognomie rührte mich nichts als sein durchdringendes Falkenauge. Mad. Calliostro stürzte mich noch tiefer hinab als ihr Mann. Mine, Ton, Bildung, Manieren u. s. w. schienen mir eine Seiltänzerin zu verrathen. Sie sprach viel von des Herrn Grafen Sparsamkeit für seinen eignen und der seinigen Leib, von seiner Aufopferung aller Freüden dieses Lebens zum besten andrer, wie er sich sogar noch niemals erlaubt habe, das Schauspielhaus (zu) besuchen, damit ja kein Leidender ungetröstet und ohn ihn zu finden aus seiner Wohnung weggehe, wie er des Nachts nur drey Stunden, ohne zu Bette zu gehen, nur in seinem Lehnsessel schlafe, länger nicht als eine Vierthelstunde an der Tafel size, wie sehr er ihren eignen Puz einschränke, um desto mehr Freigebigkeiten ausüben zu können, wie sie sich Jahr und Tage mit den gleichen Robben behelfen müsse und darum nicht in groBen Assembleen erscheine. Sie gerieth in tiefes Erstaunen über eine Robbe von seidenem Stoffe, die Mad. Sarasin ihr wies, und deren Stoff 300 Gulden kostete u. s. w. Beweis daß sie nicht gewohnt war, viel kostbare Robbes 
zu tragen! Nun consultierte meine Frau den Grafen über ihre Magenkrämpfungen, ihr gichterisches Hoquet und die Schwäche ihrer Nerven. Ich erstaunte über seine Antworten, fand in ihm nicht blo $B$ den geschikten Arzt, sondern den beobachtenden Philosophen, den tiefen Menschenkenner, den scharfsinnigen Physiognomisten, der von den äußern Würkungen zu den verborgnen Ursachen mit der größten Bestimmtheit hinaufstieg, der aus physischen Übeln die Grundanlage des Temperamentes und sogar die Hauptzüge des moralischen Carakters erklärte, und zu Heilung derselben nicht weniger kluge sittliche Verhaltungsregeln als medicinische Diät und Arzneyen vorschrieb. Ich erröthete über mein voreiliges physiognomisches Urtheil. Er gab ihr Mittel, von welchen sie zimlich gute Würkung verspürte, besuchte uns gleich Morgens darauf, gab uns Rathschläge für die bestmögliche Einrichtung unserer kleinen Haushaltung, zeigte sich uns ganz als Menschenfreünd im vortheilhaftesten Lichte u. s. w. Ich besuchte den Mann einige Male des Abends, hörte von ihm bald eine interessante Unterredung von der Verschiedenheit des Temperamentes und Carakters der Europäischen und Orientalischen Damen, wie jenne gegen diese nur Milchkinder und Puppen, diese in ihren Leidenschaften Löwinnen und Furien wären! (Freylich im Grund alles Gemeinpläze, allein mit Wiz und Laune ausgedrükt!) Vielöfters hört ich ihn aber Contes de ma Mère L'Oye, Fagots à dormir de bout und die abgeschmaktesten Garonne Prahlereyen hersagen. Ich will Ihnen, verehrungswürdiger Greis, ein paar, die ich mit eignen Ohren angehört habe, erzählen, um Sie selber zum Richter zwischen Calliostro und mir zu sezen.

Einmal behauptete er, daB er in der Türkey in einem Zeitraum von drey Monaten eilf Millionen Menschen von der Pest geheilet habe Ein andermal - daß er unbewaffnet nur von einem Bedienten begleitet im sandichten Arabien von einer zahlreichen Räuberbande wäre überfallen worden. Hierauf hätt' er sich begnügt, sie anzureden und ihnen seinen Geschlechtsnamen zu entdeken, worauf sich soglejch die ganze Bande in die Flucht begeben habe, ohn' ihm eines Hellers wehrt zu rauben. Als er mit dem Leibarzt der russischen Kayserinn einst in einen heftigen Wortwechsel gerathen, hab' er ihm einen medicinischen Zweykampf anerboten. Nemlich er sollt' in einen Becher sein stärkstes Gift, das er zu verfertigen fähig sey, gießen - Er Calliostro wolle in einen andern Becher Gift von seiner Erfindung mischen und zuerst seines Gegners Giftbecher ausleeren - der Erfolg werde zeigen, wer von ihnen der größere Chymiker seye, sein Gegner hab' es aber nicht gewagt, das Cartell anzunehmen u.s. w.

Freylich weiß ich nun wol, daß Calliostro in seiner gedoppelten Rolle als Reisender und als Arzt vor allen andern Erdensöhnen aus Brief und Siegel besizt, Windbeüteleyen auszukramen. Ob er aber durch solch handgreifliche Ammenmährchen nicht noch sein anerkanntes gedoppeltes Privilegium übertreibe, überlaß ich Ihnen, verehrungswürdiger Greis, und der ganzen unpartheyischen Welt zu beurtheilen! 
Meine Frau bediente sich ungefehr 8 bis 10 Tage lang seiner Mittel, die immer sehr gelind würkten, und in der That, seitdem sie sich in Straßburg aufhält, hat sie beynahe gar keine oder nur sehr unmerkliche Magenschmerzen empfunden. Hingegen ist sie mehr als Zeitlebens von Flüssen, Zahn- und Ohrenschmerzen geplagt, denen sie bisher niemals unterworfen war, das ich aber freylich weder auf Calliostros noch seiner Arzneyen, sondern nur auf Rechnung der drey Monate dauernden regnigten und neblichten Witterung seze. $\mathrm{Zu}$ unserer größten Verwunderung verschwand plözlich von dem Tage an, da ich ihm seine Arzneyen in dem Preis, wie er sie anschlug, bezahlt hatte, sowol Calliostro als sein Sancho Pansa, der Feldscheerer, von unsern Augen und ward niemals mehr in unserm Hause erblikt, ob er gleich täglich in unsere Nachbarschaft zu der Baroninn von Dietrich fuhr. Dieses Problem wuBt ich mir durch seine Menschenliebe ganz und gar nicht aufzulösen. Ich forschte dem Manne allenthalben genauer nach, um über seinen Carakter mehreres Licht zu bekommen, und erhielt endlich folgende Aufkiärung: 1. Magenkrämpfungen und Nervenschwächen wären für den berühmten Calliostro zu alltägliche Sak Übelkeiten, die er seiner Kunst unwürdig hielt, und die er gewöhnlichen Handwerksärzten zur Pfuscherey und zum Spielball überließ(e) - Sein Genie weyhe sich nur den Riesenkrankheiten als: Steinschmerzen, fallender Sucht, Lähmung der Glieder, Harnwinde, Miserere u. s. w., deren Heilung mit einem laut schallenden Hosianna des Pöbels begleitet sey. 2. Mög' es vielleicht meine Frau auch darinn versehen haben, daß sie der Gräfinn nicht regelmäßig genug ihre Cour gemacht, wofür der Graf sehr empfindlich sey. 3. Sey es ein nicht geringer Naturfehler gewesen, daß sich meine Frau weder Son Altesse, noch Mad. la Comtesse, noch Mad. la Marquise, noch Mad. la Baronne genennt, nicht einmal ein Von vor ihren Geschlechtsnammen zu sezen die Klugheit gehabt habe - woraus denn immer für ihn eine nur im Dunkeln schleichende Werkeltagscur entstanden wäre, die nicht einmal die Schaffhauser Zeitung ausgeposaunt hätte. Ein gleiches Schiksal hat auch einige Pariser und Lyonner Frauenzimmer, ebenfalls ohne Rang und Titul und ohne Riesenkrankheit, betroffen.

Was mir Lavaters Achtung und Freündschaft für Calliostro unerklärlich macht, ist, daB mir einige rechtschaffene Männer von hier, die anfänglich mit Calliostro in einem Ton von Vertraulichkeit standen, versicherten - Calliostro wäre ein Freygeist, der alle geoffenbarte Religion ohne Barmherzigkeit auf die Seite werfe - auch hab er bisweilen Einfälle geäußert, die sogar nach dem Atheismus röchen - und diese Männer, die ich kenne, sind weder Theologen noch strenge Orthodoxen. Nun weiß ich, daß Lavater mit ihm über religiöse Gegenstände eingetretten ist, da er das erstemal in Straßburg war, $\left.{ }^{1}\right)$ und daß er sich her-

1) Lavaters Tagebuch 1781: "Jenner 22 auf Basel zu Sarasin. 23 auf Straßburg. 24 und 25 meistens bei Calliostro. C. prätenđiert, mit den höchsten himmlischen Geistern zuweilen im Umgang zu stehen." 
nach in Zürich über sein Glaubensbekenntnis nicht unzufrieden bezeigte. Nun von zweyen eins - Entweder war Calliostro gegen Lavatern Heüchler - oder - oder Lavater dehnt seine Duldsamkeit weit stärker aus, als ich - und vielleicht auch Sie vermuthet hätten. So viel von Calliostro nun, verehrungswürdiger Greis - nur noch ein paar Fragen. Will Calliostro mehr nicht seyn als ein Weiser, ein großer Arzt und uneigennüziger Wolthäter der leidenden Menschheit, wofür der eitle Titul des Grafen von? - der so manchen Dummkopf zieret - da er doch jedem, der es hören will, gesteht, daß der Namme Graf von Calliostro nur ein Nom de Guerre, nicht sein wahrer Name sey, welchen letztern er sorgfältig verheelet. Ist dem Philosoph oder auch dem bloß vernünftigen Manne der Graf von schätzbarer als der Weise, der große Arzt, der Wolthäter Calliostro tout court? Wozu die drey Bedienten in grün mit silber verbrämter Livree, die Crystallleüchter, die damastenen Vorhänge usw. Wozu sein entschiedener Hang zu den Großen dieser Welt, auf deren Ordensband und Stern er (im vertraulichen Tête à Tête) spuken zu wollen vorgiebt? Sich ihren Schuz und Gunst zu erwerben! Wozu bedarf er diese, so lang er die Ruhe, das Glük der menschlichen Gesellschaft eher befördert als störet? Überla $\beta$ er dieses seinen auszeichnenden Geistesgaben, seiner GroBmuth und Menschenliebe! Wozu die Ammen Mährchen und Calender Geschichten, worinn immer sein theüres Ich die Haubtrolle spielt, den großen Haufen zu blenden und an seinen Triumphwagen zu fesseln? Nun was ligt denn dem Weisen, dem großen Arzt, dem Wolthäter der Menschheit am Anstaunen, an der Bewunderung des großen und kleinen Pöbels Sollte er nicht vielmehr über solchen Beyfall erröthen?

Sehr oft hab' ich hier Calliostros Simplicität und natürliches Wesen rühmen hören - und in Gottes Namen ich kann diese Eigenschaften, meiner Aufmerksamkeit auf ihn ungeachtet, nirgendswo als in seiner Kleidung, Haarpuz, in der Flegeley seines Tones und Manieren und in seinem geradebrechten italiänisch-französischen Galimathias finden. Oft hab' ichs versucht, ihn mit dem hochseligen Micheli Schuppach ${ }^{1}$ ) zu vergleichen - aber welch himmelweite Kluft ist zwischen diesen beyden Männern bevestigt! Das Potpourri und Millefleurs der Abendkränzgen in Calliostros Hause, und die Tischgesellschaft in Langnau - Calliostros Grafenstand, drey Bediente, Crystallleüchter, und Hôtel à Porte-Cochère, und Michelis Nürenberger Kupferstiche, womit alle Wände beklebt waren, seine unzählbaren Vogelkefigte, wächserne Männchen u. s. w. mögen einige Ähnlichkeiten haben. Aber Mann für Mann - Micheli hatte die wahre Simplicität eines Alpenbewohners und ward so zusagen Médecin sans le savoir - Calliostro die erkünstelte Simplicität eines verschlagenen Charlatans. Micheli sagte pfeifend: Mir wey öpe lugen, was anzstellen syge es wird öpe nit so grüselig fählen. Calliostro sagt, die Hand auf die Brust

1) Michael Schuppach (gen. Micheli) Bauer und Wunderdoktor zu Langnau im Emmental, der aus ganz Europa Besuche empfing. 
legend: Le Comte de Calliostro Vous répond de Vous guérir. Micheli gieng in seinen Arzneyen piano und heilte langsam - nuzten seine Arzneyen nichts, so schadeten sie nichts. Calliostro spielt bey seinen Patienten meistens Va la Banque und macht quitt ou double. Um gerecht zu seyn, muß ich gestehen, daß ich Calliostro weit mehr Theorie in seiner Kunst zutraue als Micheli - vielleicht $\mathrm{da} B$ er nicht vielmehr darüber gelesen und ex professo studiert, aber unendlich mehr gesehen, erfahren und beobachtet hat, mit größern Anlagen geboren war als Micheli, sich in Situationen befand, wo er sie besser entwikeln konnte. Größerer Arzt scheint mir Calliostro unstreitig - lieber wäre mir Micheli als Mensch.

Verzeyhen Sie, verehrungswürdiger Greis, unvermerkt ist unter meinen Händen mein freündschaftlicher Brief zur diken Epistel angeschwollen - doch ich unterhielt mich mit Ihnen, und mein Süjet schien mir interessant. Nach und nach erhol ich mich von meiner tiefen Verehrung für weltberühmte Männer. Bald scheinen sie mir wie unsere hohen Alpgebürge, die sich in der Ferne in ihrem schönsten Glanze spiegeln und nahe frostige Eismassen oder öde Felsenwände sind. Sie und Geßnern werd' ich immer in der Nähe und in der Ferne verehren! - weil Sie nicht bloß berühmte - nein auch große Männer sind.

Übrigens schrieb ich diesen Brief nur Ihnen, verehrungswürdiger Greis, und wünschte sehr, daB nichts davon weder Lavatern noch irgend einem glühenden Lavaterianer noch auch Herrn Professor Breitinger zu Gesichte oder zu Ohren käme. Beyde sind Herrn Sarasins Busenfreünde, der sich auch hier aufhält, und noch viel weniger möcht ich in einem fremden Lande als in meinem eignen Vaterlande in eine medicinischlitterarische Fehde verwikelt werden, wovon ich nichts verstehe. Werden doch alle Lappereyen, die der Zürcherische Abderitismus tagtäglich hervorkeimen läßt, siedendwarm nach Straßburg überschrieben, und ich gesteh' es Ihnen, ich verehre die Quelle, woraus Sarasins Schwermerey und Anbetung für Calliostro fließt. Hätt' er meiner Frau das Leben gerettet, wie es ihm und seiner Kunst Mad. Sarasin zu danken hat, hätt' er meinem Sohne ${ }^{1)}$ Gesundheit und Kräfte geschenkt, die ihm eine gichterische Nervenkrankheit beynahe gänzlich geraubt hatte, und die kein anderer Arzt heilen konnte - ich fühl' es, unmöglich könnt' ich von ihm mit Gleichgültigkeit reden. -

1) Am 21. Mai 1781 hatte Sarasin auch seinen Sohn Felix in Cagliostros Heilbehandlung gegeben. Auch diese Kur war erfolgreich. 